\title{
COMPUTER SIMULATION OF A SOLAR MICROWAVE BURST
}

\author{
G. Bruggmann, E. Schanda, A. Magun \\ University of Bern, switzerland
}

\section{INTRODUCTION}

A simple impulsive event observed at microwave frequencies between 3.2 and $50 \mathrm{GHz}$ is simulated with a homogeneous source model. Intensity and polarization of the 1984 April 26 event are shown in fig. $l a$ and $1 b$, respectively. The spectral maximum is close to the observing frequency of $11.8 \mathrm{GHz}$.

The time profiles of the intensity I and the circular polarization $\rho_{c}$ at this frequency as well as the spectrum at different characteristic phases (rise, maximum and decay) are calculated and compared with the observation in order to deduce parameters of the microwave source.

INSIITUTE OF RPPLIEO PIISSICS. UHIYERSTIT OF BERH. SHITZEMLAND

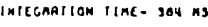

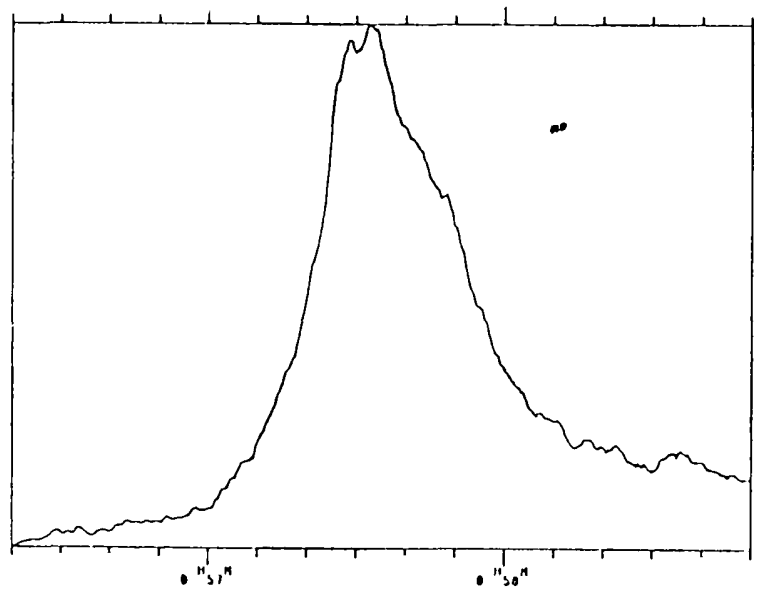

Ut ON MP. 20 1904

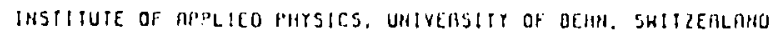
Imiganilon rinc. 304 hs

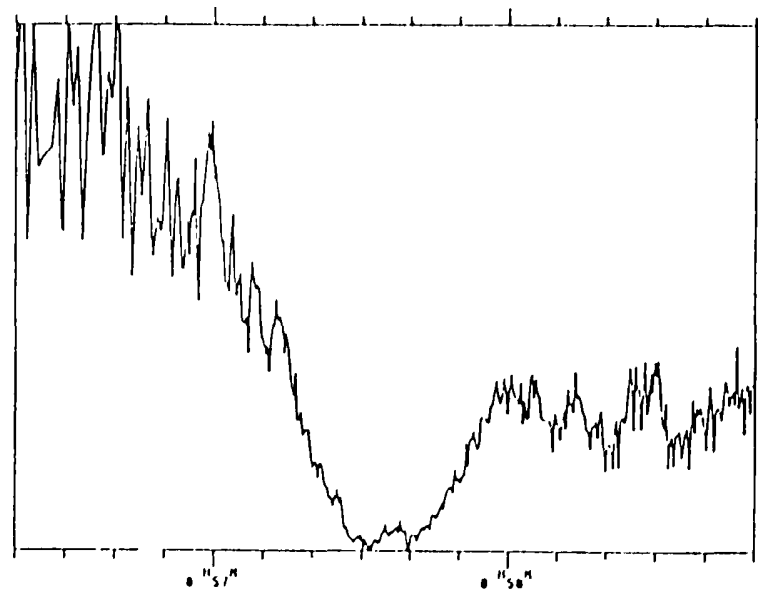

IT ON APR. 261304

Fig. 1 Observed Intensity (a) and Polarization (b) at $11.8 \mathrm{GHz}$ scales: 22 - $334 \mathrm{SFU}$ (a) and $14 \%$ - $34 \%$ (b)

\section{SIMULATION}

The emission formulas are based essentially on Ramaty (1969) calculating the gyrosynchrotron radiation from an ensemble of electrons with homogeneous density and a power law energy 
spectrum. Radiative transfer is assumed to take place in an ambient medium of homogeneous density and magnetic field.

Magnetic field: 400 gauss

Plasmafrequency: $500 \mathrm{MHz}$

$\begin{array}{ll}\text { Source area: } & 25 \text { arcsec }^{2} \\ \text { Aspect angle: } & 75 \mathrm{o}^{2}\end{array}$

The electron spectrum is characterized by an isotropic pitch angle distribution and a power law energy spectrum:

$$
\mathrm{N}(\mathrm{E})=\mathrm{KE}^{-\delta}
$$

$\delta$ is the spectral index and

$$
K=N_{L} A(\delta-1) / E_{0}^{1-\delta}
$$
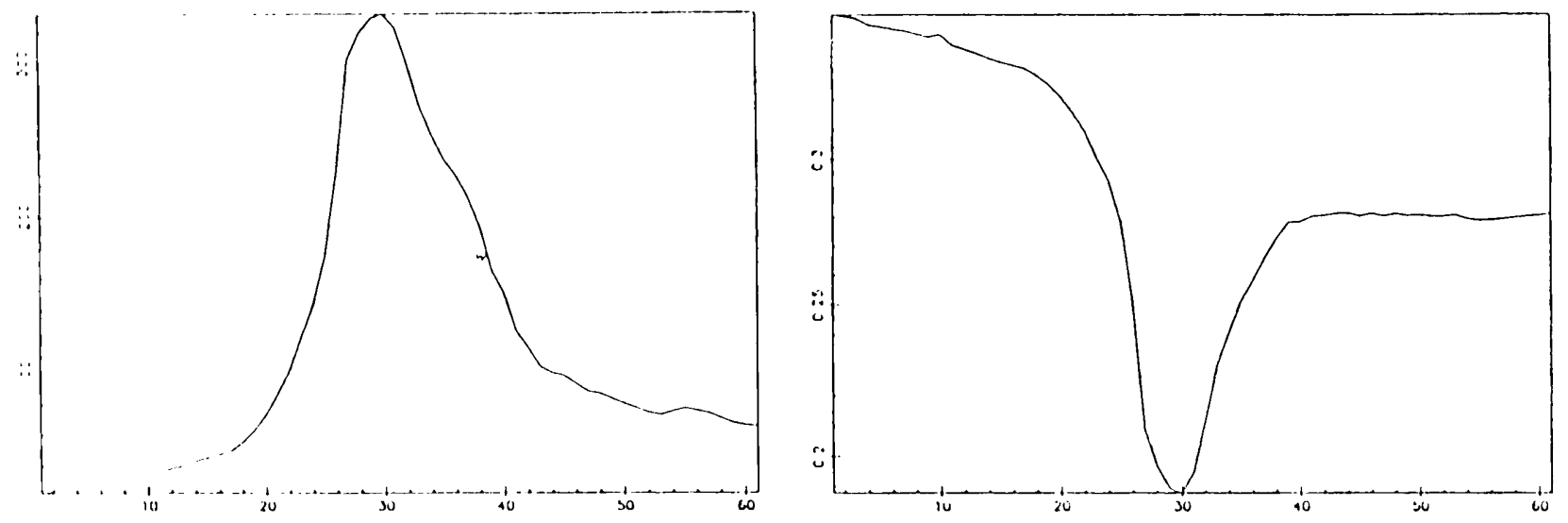

Fig. 2 simulated Intensity $I(t)$ and polarization $\rho_{\mathrm{C}}(t)$ at $11.8 \mathrm{GHz}$ 1 time step corresponds to $2.5 \mathrm{sec}$.
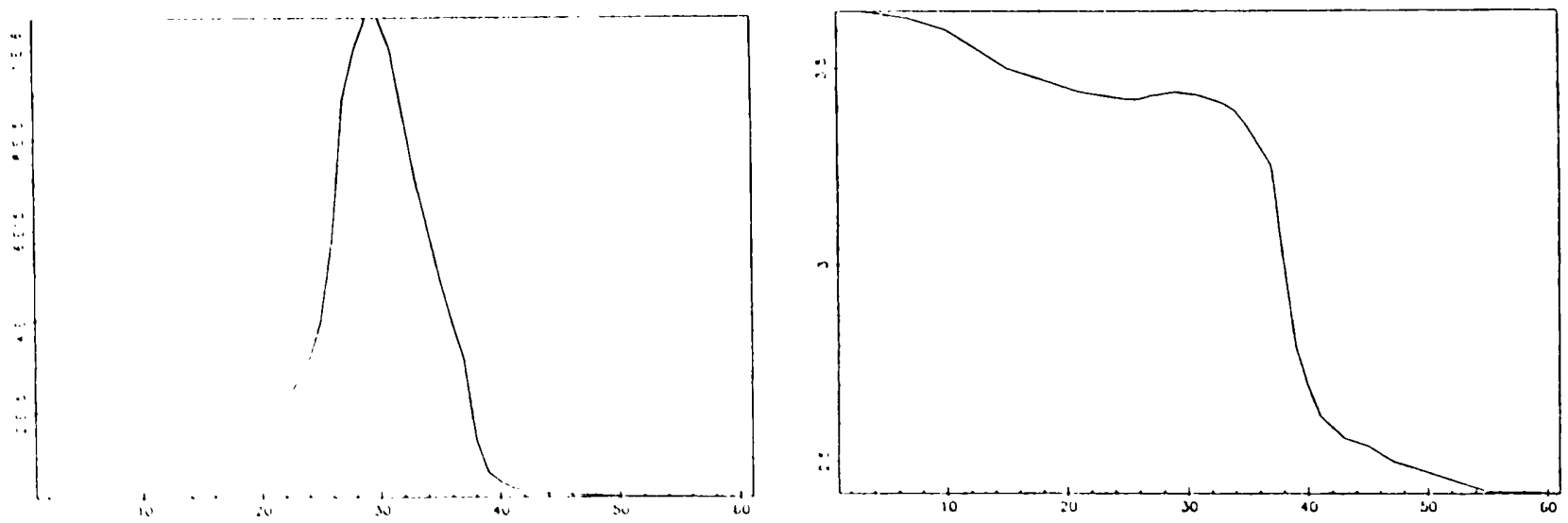

Fig. 3 Best fit column density $N_{L}(t)\left[\mathrm{cm}^{-2}\right]$ and spectral index $\delta(t) .1$ time step corresponds to $2.5 \mathrm{sec}$.

$A$ is the constant source area and $E_{0}=25 \mathrm{keV}$ is a constant low energy cutoff. The column density $\mathrm{N}_{\mathrm{L}}$ is proportional to the total number of nonthermal electrons and is one of the two independent 
source parameters varying throughout the event. The other variable is the spectral index $\delta$.

The intensity and polarization curves of $f i g .2 a$ and $2 b$ are calculated point by point with source parameters $N_{L}(t)$ and $\delta(t)$ according to fig. $3 a$ and $3 \mathrm{~b}$. They represent the best fit of observations with simulations.

Fig. 4 finally compares the observed and calculated microwave spectrum at different characteristic phases of the event (rise, maximum and decay phase).
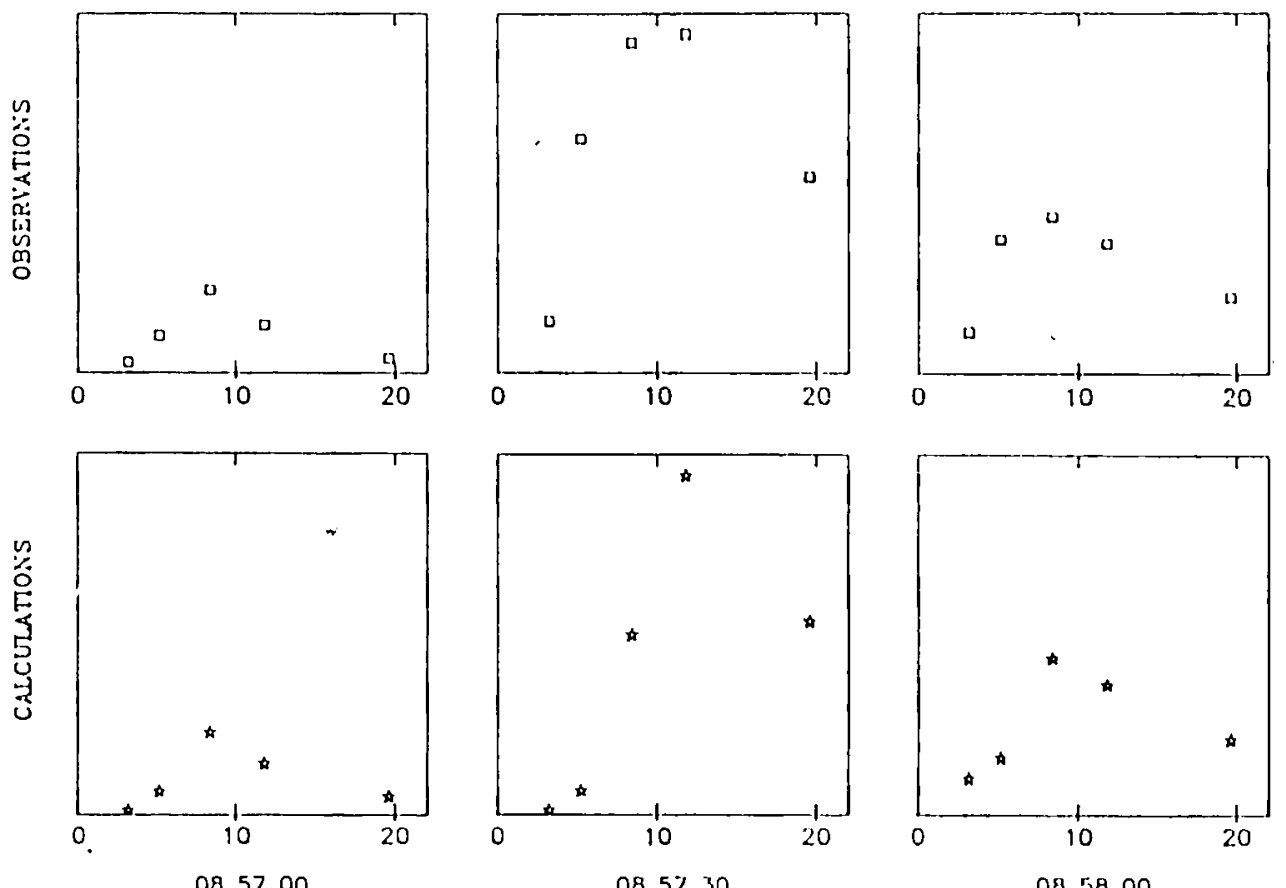

085800

Fig. 4 observed (top) and simulated (bottom) spectra at three characteristic phases (rising phase, maximum and decay phase). Linear intensity scale: 0 - 350 SFU

\section{DISCUSSION}

A measure for the deviation between the abserved parameter $P_{\text {obs }}$ and the calculated parameter $P_{\text {sim }}$ is given by the mean value of

$$
\left(\mathrm{P}_{\text {obs }}{ }^{-P_{\text {sim }}}\right) / \mathrm{P}_{\text {sim }}
$$

and the corresponding standard deviation. In these terms, the intensity is simulated with an accuracy of $0 \%+1-4 \%$, while the deviation in polarization is given by $-27 \%+1-12 \%$.

An explanation for the systematic $38 \%$ deficiency of the observed polarization might be mode coupling (Zhelezniakov and zlotnik, 1964). Partial depolarization can occur if the radiation passes an area of transverse magnetic field. on the other hand, the existence of two microwave sources radiating at the same time with opposite sense of polarization could also produce the same effect. 
Inspite of the very simple assumptions, a good agreement was found between the simulation and the observations.

A comparison with the electron energy spectrum derived from hard $x$-ray data (Dennis, 1986) yields interesting results: The temporal behavior of the total number of electrons needed to produce the hard $x$-rays and the microwaves is approximately the same. For a thick target model, the number of electrons is about three times smaller and for a thin target model about 20 times smaller than derived from our simulation.

However the spectral index according to the hard $x$-rays does not show a decrease after the intensity maximum, but stays constant around 5.3 for the thick target model and 3.8 for the thin target model.

Obviously, the electron spectrum after the intensity maximum derived from hard $x$-rays does not fit the distribution derived from the microwave simulation. There might be several explanations:

- The decreasing spectral index may be due to trapping of electrons in the microwave source (Vilmer, 1985 ).

- The electron populations producing the microwaves and the hard $x$-rays are not identical.

- The simple assumption of the time independant source parameters might not be correct. Especialiy changes in source area or magnetic field strength may be important.

\section{REFERENCES}

DENNIS, B.: private communication, 1986

RAMATY, R.: Astrophys. J. 158, 753, 1969

VILMER, N.: thesis, University of Paris, France, 1985

ZHELEZNYAKOV, V.V.: Soviet Astron.-AJ7, 485, 1964 